\title{
AVALIAÇÃO DA FORÇA MÁXIMA ISOMÉTRICA DE ROTADORES INTERNOS E EXTERNOS DO OMBRO DE TENISTAS JUVENIS
}

\author{
Carlos Eduardo Campos \\ Universidade de Itaúna \\ Programa de Pós-graduação em Ciências do Esporte UFMG \\ Paulo Eduardo Souza Medeiros \\ Universidade de Itaúna \\ paulofisic@yahoo.com.br \\ Crislaine Rangel Couto \\ Programa de Pós-graduação em Ciências do Esporte UFMG
}

Cláudio Lúcio Marini Cardoso

Centro Universitário de Belo Horizonte-MG

André Gustavo Pereira de Andrade

Programa de Pós-graduação em Ciências do Esporte UFMG

Recebido em: 18/12/2014

Aprovado em: 08/15/2015

\section{RESUMO}

Vários estudos com atletas que utilizam a cintura escapular de forma específica foram publicados nos últimos anos, indicando um desequilíbrio muscular na articulação do ombro. Esse desequilíbrio muscular poderia causar lesões nessa articulação. $\mathrm{O}$ assunto e as abordagens a respeito vêm gerando um interesse crescente. $\mathrm{O}$ objetivo foi quantificar os valores de força máxima isométrica dos rotadores internos e externos do ombro, bem como determinar a diferença de força entre o membro dominante e o não dominante. A amostra foi composta por 9 tenistas de competição em nível estadual, todos do sexo masculino de uma equipe juvenil com idade variando entre 15 a 18 anos. Foi utilizado um equipamento que se constitui de uma mesa com um suporte giratório para mensurar a força máxima isométrica dos rotadores internos e externos do ombro e uma célula de carga com capacidade de $500 \mathrm{kgf}$. Os testes foram bilaterais, com 3 tentativas para cada movimento e com 5 segundos de duração. Foi identificada diferença significativa $(\mathrm{P} \leq 0,05)$ entre os valores médios da força máxima isométrica dos

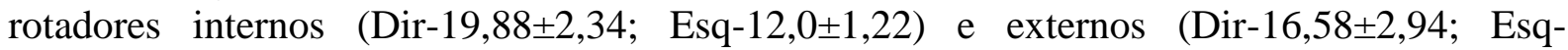
$11,23 \pm 1,90$ ) do ombro e também em relação ao membro dominante (ombro direito)quando comparado com o não dominante (ombro esquerdo). Os resultados encontrados netse estudo mostram uma diferença entre os valores da força máxima isométrica de rotadores internos e externos dos membros superiores e uma maior força do membro dominante quando comparada com os valores do membro não dominante.

Palavras-chave: Rotadores de ombro. Força isométrica. Jogadores de tênis. 
CAMPOS, C. E.; MEDEIROS, P. E. S.; COUTO, C. R.; CARDOSO, C. L. M.; ANDRADE, A. G. P. de. Avaliação da força máxima isométrica de rotadores internos e externos do ombro de tenistas juvenis

\title{
ASSESSMENT OF MAXIMUM ISOMETRIC STRENGTH OF INTERNAL AND EXTERNAL ROTATORS OF SHOULDER OF YOUTH TENNIS PLAYERS
}

\begin{abstract}
Several studies with athletes who use the shoulder girdle in a specific manner have been published in recent years, indicating a muscle imbalance in the shoulder joint. This muscle imbalance could cause injuries in this joint. The subject and approaches have created a growing interest.The objective was to quantify the values of maximum isometric strength of internal and external rotators of the shoulder and to determine the difference in strength between the dominant and non-dominant limb. The sample consisted of 9 tennis players of competition at the state level, all male from a youth team, aged between 15 to 18 years. An equipment that consists of a table with a swivel support to measure the maximum isometric strength of internal and external rotators of the shoulder, and a load cell with a capacity of $500 \mathrm{kgf}$. The tests were bilateral with 3 attempts for each movement and with 5 seconds of duration. It was detected a significant difference $(\mathrm{P} \leq 0.05)$ between the average values of maximum isometric strength of the internal rotators (Right-19.88 \pm 2.34 ; Left-12.0 \pm 1.22 ) and external rotators (Right-16.58 \pm 2.94 ; Left- $11.23 \pm 1.90$ ) of the shoulder and also relative to the dominant limb (right shoulder) when compared with the non-dominant (left shoulder). The results found in this study show a difference between the values of maximum isometric strength of internal and external rotators of the upper limb and greater strength of the dominant limb compared with values of the nondominant.
\end{abstract}

Keywords: Shoulder rotators. Isometric strength. Tennis players.

\section{INTRODUÇÃO}

A força muscular é um importante componente da aptidão física relacionada à saúde ${ }^{1} \mathrm{e}$ ao desempenho atlético (KNUTTGEN; KOMI, 2006), especialmente no tênis, atividade que deve ser desenvolvida de uma forma específica para que o jogador obtenha o máximo rendimento possível dentro de suas possibilidades (CHANDLER et al, 1992). Vários estudos com atletas de tênis, beisebol, natação e pólo aquático foram publicados, indicando um desequilíbrio muscular na articulação do ombro, assunto que vem gerando um interesse crescente (ELLENBECKER et al, 2007; ELLENBECKER; MATTALINO, 1997; HILL; PRAMANIK; MCGREGOR, 2005; MCMASTER et al, 1991). Essa alteração é significativa no ombro de atletas, quando comparada a não atletas, e demonstra um fortalecimento excessivo dos rotadores internos (RI) em relação aos rotadores externos (RE) e dos adutores em relação aos abdutores do ombro. Para McMaster et al (1991) atletas que são submetidos a treinamentos de rotação interna com maior incidência, estão sob risco duplo, pois já existe uma diferença natural entre a força de rotadores internos e externos. Isso foi demonstrado por Sahrmann 
CAMPOS, C. E.; MEDEIROS, P. E. S.; COUTO, C. R.; CARDOSO, C. L. M.; ANDRADE, A. G. P. de. Avaliação da força máxima isométrica de rotadores internos e externos do ombro de tenistas juvenis

(2005) que mostra essa diferença de força dos rotadores internos e rotadores externos do ombro e que pode ser explicada pelo tamanho e número de músculos responsáveis pela rotação interna.

Esse desequilíbrio pode desencadear mecanismos de lesões musculares, tendinosas ou articulares (ANSENJO, 1998; CAMPOS et al, 2004), comprometendo o rendimento dos atletas. Nessa perspectiva, Hamill e Knutzen (2008) afirmam que a articulação do ombro está mais suscetível a lesões em esportes que envolvem movimentos do braço abduzidos acima de noventa graus.

A maioria dos estudos que investigaram essa diferença de força dos rotadores internos e externos do ombro utilizou testes laboratoriais de alta tecnologia, com custo financeiro elevado e de difícil acesso para a população atlética. Por esse motivo, a utilização de um teste que seja de fácil aplicação, baixo custo e com confiabilidade comprovada (CAMPOS et al, 2007) pode ser importante para fornecer subsídios aos profissionais da área de saúde.

Portanto, o objetivo deste estudo é quantificar os valores da força máxima isométrica dos RI e os RE dos membros superiores, bem como determinar a diferença de força entre membro dominante e não dominante de jogadores de tênis de quadra.

\section{METODOLOGIA}

A amostra foi composta por 09 tenistas de competição em nível estadual, do gênero masculino, de uma equipe juvenil, com idade variando entre 15 e 18 anos.

Inicialmente, foram determinadas a massa corporal, estatura, as circunferências de braço e antebraço e as dobras cutâneas. Para medição antropométrica das circunferências dos segmentos corporais, foi utilizada uma fita métrica Sanny e um plicômetro Skinfold Caliper; uma balança Filizola, para medição da massa corporal total; e um estadiômetro Filizola, para medição da estatura.

Para a medição da força isométrica máxima nos rotadores internos e externos foi utilizada uma célula de carga da marca Líder com capacidade para 500 kgf e uma mesa rotadora (Fig. I) baseada no estudo de Souza (2003). Esse equipamento constitui-se de uma mesa com um suporte giratório (a) para o apoio do ombro e punho; para fixação da mão era usado um manípulo (b). Paralelamente ao punho de fixação da mão e no mesmo alinhamento em um braço giratório é acoplada uma célula de carga (c). No braço giratório é afixada uma fita métrica (d) rente ao apoio do punho, para que a mesma marcação individual seja feita tanto para o braço direito quanto para o braço esquerdo. Para garantir maior estabilidade da ação, o antebraço era fixado com uma fita. De acordo com estudo de Campos et al (2007) o equipamento citado 
CAMPOS, C. E.; MEDEIROS, P. E. S.; COUTO, C. R.; CARDOSO, C. L. M.; ANDRADE, A. G. P. de. Avaliação da força máxima isométrica de rotadores internos e externos do ombro de tenistas juvenis

apresenta resultados confiáveis para determinação da força isométrica dos RE e RI $(r=0,956)$. Esses resultados se aproximam aos de Kolber et al (2007) que utilizaram um dinamômetro isométrico com um método semelhante ao utilizado neste estudo e encontraram uma confiabilidade de $r=0,972$.

Figura 1 - Mesa rotadora para medição da força isométrica (baseada no estudo de SOUZA, 2003 - ver ANEXO 1)

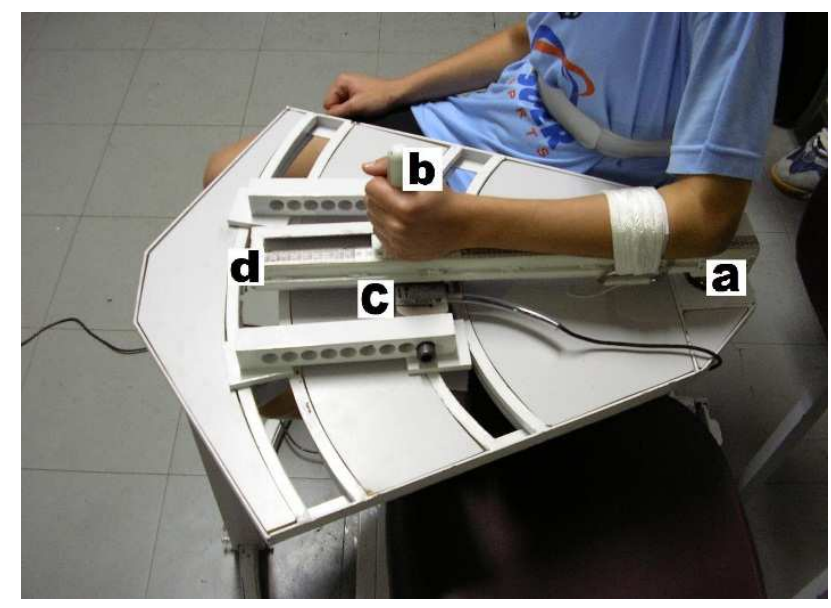

Para o procedimento de medição, os avaliados foram encaminhados para a mesa rotadora. Logo após, os indivíduos sentavam na cadeira do aparelho e era medido o comprimento do antebraço para a fixação da mão no manípulo. Os avaliados foram orientados a realizar duas tentativas submáximas antes da medida definitiva da força, com o objetivo de adaptação ao teste. A medição da força isométrica máxima dos rotadores do ombro era iniciada seguindo os seguintes procedimentos: (1) sentado com tronco ereto apoiado no encosto na cadeira; (2) braço abduzido a 10 graus; (3) cotovelo apoiado a 90 graus de flexão; (4) antebraço em posição neutra; (5) mão fixada ao manípulo; (6) ombro no plano da escápula, ou seja, 30 a 45 graus anteriores ao plano frontal. Os indivíduos foram orientados a não fazer nenhuma postura compensatória indesejável (flexão lateral do tronco ou rotação do tronco).

Cada sujeito foi submetido aos testes bilateralmente, realizando 3 (três) tentativas de rotação externa e de rotação interna, com duração do estímulo de 5 (cinco) segundos e intervalo de 2 (dois) minutos entre as tentativas (BADILLO; AYESTARÁN, 2001). A ordem do lado a ser testado foi aleatória. Após as três tentativas, a cadeira era trocada de lado e o mesmo procedimento era adotado com o outro membro. Todos os indivíduos foram verbalmente encorajados a manter a contração e o esforço máximo foi enfatizado. 
CAMPOS, C. E.; MEDEIROS, P. E. S.; COUTO, C. R.; CARDOSO, C. L. M.; ANDRADE, A. G. P. de. Avaliação da força máxima isométrica de rotadores internos e externos do ombro de tenistas juvenis

A análise dos dados foi realizada através do programa estatístico SPSS ${ }^{\circledR}$ versão 11.0 for Windows ${ }^{\circledR}$. Foi utilizada a análise descritiva para caracterização da amostra e da força muscular. Para a comparação do rendimento da força muscular entre membros dominantes e não dominantes, foi utilizado o teste t para amostras emparelhadas. E para verificar a normalidade da amostra foi utilizado o teste Kolmogorov-Smirnov.

Antes da realização dos testes, os participantes receberam um termo de consentimento livre e foram esclarecidos sobre os objetivos da pesquisa. Para os menores de 18 (dezoito) anos, houve autorização dos pais ou responsáveis para a participação e a utilização dos dados pelo pesquisador, sendo respeitado o anonimato.

\section{RESULTADOS}

Tabela 1 - Caracterização antropométricas da amostra $(n=9)$

\begin{tabular}{cc}
\hline Variáveis antropométricas & Média e Desvio Padrão \\
\hline Estatura & $174,56 \pm 4,69 \mathrm{~cm}$ \\
Gordura & $12,65 \pm 1,81 \mathrm{~kg}$ \\
Massa magra & $61,72 \pm 5,28 \mathrm{~kg}$ \\
\hline
\end{tabular}

Tabela 2 - Comparação entre as médias da força dos músculos rotadores internos e externos do ombro $(n=9)$

\begin{tabular}{lcc}
\hline & Média (kgf) e Desvio Padrão & Nível de Significância \\
\hline RI dir & $19,88 \pm 2,34$ & 0,00 \\
RE dir & $12,01 \pm 1,22$ & \\
RI esq & $16,59 \pm 2,94$ & 0,00 \\
RE esq & $11,23 \pm 1,90$ & \\
\hline $\boldsymbol{p} \leq \mathbf{0 , 0 5}$ & \\
Legenda: RI dir - Média de rotadores internos do braço direito \\
RE dir - Média de rotadores externos do braço direito \\
RI esq - Média de rotadores internos do braço esquerdo \\
RE esq - Média de rotadores externos do braço esquerdo \\
Kgf - quilograma força
\end{tabular}

A TAB. 2 mostra os valores da média, desvio padrão e nível de significância, da força máxima isométrica de rotadores internos e externos dos membros superiores. Os dados indicam 
CAMPOS, C. E.; MEDEIROS, P. E. S.; COUTO, C. R.; CARDOSO, C. L. M.; ANDRADE, A. G. P. de. Avaliação da força máxima isométrica de rotadores internos e externos do ombro de tenistas juvenis

que houve diferença significativa entre os valores médios da força máxima isométrica de rotadores internos e rotadores externos dos membros superiores.

Tabela 3 - Diferença entre as médias da força dos músculos rotadores internos e externos do ombro dominante e não dominante $(n=9)$

\begin{tabular}{lccc}
\hline & Média (kgf) e Desvio Padrão & Nível de Significância \\
\hline Par 1 & RID & $19,87 \pm 2,34$ & 0,02 \\
& RIND & $16,58 \pm 2,94$ & \\
Par $\mathbf{2}$ & RED & $12,00 \pm 1,22$ & 0,05 \\
& REND & $11,23 \pm 1,90$ & \\
\hline $\boldsymbol{p} \leq \mathbf{0 , 0 5}$ & & \\
Legenda: RID - Média de rotadores internos do braço direito & \\
RED - Média de rotadores externos do braço direito & \\
RIND - Média de rotadores internos do braço esquerdo & \\
REND - Média de rotadores externos do braço esquerdo &
\end{tabular}

A TAB. 3 mostra os dados da comparação do rendimento entre o membro dominante e o não dominante através do teste t simples. Eles indicam que houve diferença significativa nos valores da força máxima isométrica de rotadores internos dominantes com rotadores internos não dominantes. E houve, também, diferença significativa entre os valores de rotadores externos dominantes e rotadores externos não dominantes.

\section{DISCUSSÃO}

O tênis é uma modalidade esportiva que expõe os atletas a grandes exigências de forma unilateral. Essa exigência desencadeia um desequilíbrio muscular do ombro dominante em relação ao não dominante. Carroll et al (2006) evidenciou um aumento da força do membro contra-lateral associada ao treinamento. Mas, isso não é suficiente para diminuir ou estabilizar o desequilíbrio causado por modalidades como o tênis. Os dados da tabela II apresentam diferenças significativas da força dos RI quando comparados com os RE, tanto para o braço direito (RI-19,8 $\pm 2,3$, RE-12,1 $\pm 1,22$ ) quanto para o esquerdo (RI-16,59 $\pm 2,94$, RE-11,23 $\pm 1,90$ ). Esses resultados vão de encontro aos achados de Treiber et al (1998), quando avaliaram a força do ombro de 22 tenistas colegiais com idade média de 21,2 anos e encontraram valores significativamente maiores da força de RI quando comparados com a RE. De forma semelhante, 
CAMPOS, C. E.; MEDEIROS, P. E. S.; COUTO, C. R.; CARDOSO, C. L. M.; ANDRADE, A. G. P. de. Avaliação da força máxima isométrica de rotadores internos e externos do ombro de tenistas juvenis

no estudo de Forgirini et al (2007), em que participaram 40 jogadores de tênis Junior (26 mulheres e 14 homens), com idade média de 14 anos, a força encontrada foi maior para os rotadores internos, tanto para o membro dominante quanto para o não dominante. Essa diferença pode ser explicada, segundo os autores, por um maior fortalecimento dos rotadores internos, visto que, durante o treinamento e as competições, são mais exigidos nos movimentos de saque e fourehand. Para Sahrmann (2005), Kibler e Safran (2000) e Doneux et al (1998) esse desequilíbrio muscular entre os rotadores internos e externos do ombro pode causar a "síndrome dos rotadores internos do ombro", onde os rotadores internos predominam sobre os rotadores externos. Essa síndrome é caracterizada pela alteração do comprimento do grande dorsal e o peitoral maior que se encontram encurtados e como sinais associados o músculo deltóide e os rotadores externos também podem estar encurtados. Nessa síndrome, há uma alteração da força muscular dos músculos rotadores externos e subescapular, que se encontram fracos, podendo causar: tendinopatia do manguito rotador, impactação do ombro, ruptura parcial do manguito rotador, tendinopatia bicipital, tendinopatia do supra espinhoso, bursite, dor na articulação acromioclavicular e tendinopatia calcificada.

Os resultados da tabela III mostram a comparação da força dos rotadores dos membros dominantes e não dominantes. Os resultados mostram valores, significativamente, maiores para a força dos rotadores internos e externos dominantes $(19,87 \pm 2,34,12,0 \pm 1,22)$ quando comparados com os rotadores internos e externos não dominantes $(16,58 \pm 2,94,11,23 \pm 1,90)$. Esses resultados corroboram com o estudo de Julienne et al (2007), que avaliou 16 atletas com idade entre 21 e 24 anos. Foram encontrados picos de toque maiores para os rotadores internos e externos do membro dominante quando comparados com o lado não dominante. A diferença de força entre os rotadores internos dominantes e os rotadores internos não dominantes pode ocorrer pelo fato de a maioria dos golpes dos tenistas, executados durante treinos ou competições, exigirem uma maior intensidade nas ações de rotação interna do ombro dominante. Essa utilização com maior frequência do membro dominante pode gerar, de acordo com Torres et al (2009), um déficit de rotação interna e esse déficit pode causar uma instabilidade da glenoumeral e um consequente desencadeamento da síndrome do impacto. Para Bernard, Codine e Minier (2004) e Brasseur et al (2004), o uso excessivo do braço dominante está relacionado com as lesões do supra espinhoso pelo fato de uma maior demanda dessas estruturas, durante os treinamentos e competições. Portanto, é necessário que seja realizado um treinamento para diminuir essa dominância entre os rotadores do ombro, visto que a própria modalidade desencadeia essa diferença. 
CAMPOS, C. E.; MEDEIROS, P. E. S.; COUTO, C. R.; CARDOSO, C. L. M.; ANDRADE, A. G. P. de. Avaliação da força máxima isométrica de rotadores internos e externos do ombro de tenistas juvenis

\section{CONCLUSÃO}

A partir dos resultados obtidos na análise estatística dos dados da pesquisa, conclui-se que houve diferença significativa entre os valores da força máxima isométrica de rotadores internos e externos dos membros superiores e do ombro dominante quando comparados com os valores do ombro não dominante.

Os resultados mostram também que o desequilíbrio muscular está presente na articulação do ombro de todos os atletas de tênis que participaram dessa pesquisa. É importante estar atento a essas ocorrências para que se busque uma forma de prevenção das lesões causadas por esses desequilíbrios.

\section{REFERÊNCIAS}

ASENJO, J. A. A. Preparación física en el tenis: la clave del éxito. Madrid: Gymnos, 1998.

BADILLO, J. J. G.; AYESTARÁN, E. G. Fundamentos do treinamento de força: aplicação ao alto rendimento desportivo. 2. ed. Porto Alegre: Artmed, 2001.

BRASSEUR, J. L. et al. Ultrasonographic rotator changes in veteran tennis player: the effect of hand dominance and comparison whit clinical findings. European Radiology, v. 14, p. 857-864, 2004.

CAMPOS, C. E.; PIRES, F. O.; GUSTAVO, A. Medição de força máxima isométrica dos rotadores internos e externos do ombro. In: CONGRESSO IBEROAMERICANO DE ENGENHARIA MECÂNICA, 8., 2007, Cusco. Anais... Cusco: FIIM, 2007.

CHANDLER, T. J. et al. Shoulder strength power, and endurance in college tennis players. The American Journal of Sports Medicine, v. 20, p. 455-7, 1992.

ELLENBECKER, T. S. Shoulder internal and external rotation strength and range of motion of highly skilled junior tennis player. Isokinetics and Exercise Science, v. 2, p. 65-72, 1992.

FORGIARINI, M. et al. Concentric and eccentric isokinetic strength profile shoulder rotators in elite juniors tennis players. In: INTERNATIONAL SYMPOSIUM ON BIOMECHANICS IN SPORTS, 25., 2007, Ouro Preto. Anais... Ouro Preto: ISBS, 2007.

HAMILL, J.; KNUTZEN, K. M. Bases biomecânicas do movimento humano. São Paulo: Manole, 2009.

JULIENE, R. et al. Isokinetic and eletromyografhic study of internal and external rotator muscles of tennis player. Isokinetics and Exercise Science, v. 15, n. 15, p. 173-182, 2007. 
CAMPOS, C. E.; MEDEIROS, P. E. S.; COUTO, C. R.; CARDOSO, C. L. M.; ANDRADE, A. G. P. de. Avaliação da força máxima isométrica de rotadores internos e externos do ombro de tenistas juvenis

KOLBER, M. J. et al. The realibity of hand-held dynamometry in measuring isometric strength of the shoulder internal and external rotator musculature using stabilization device. Physiotherapy Theory and Practice, v. 23, n. 2, p. 119-124, 2007.

KOMI, P. V. Força e potência no esporte. Porto Alegre: Artmed, 2006.

MCMASTER, W. C.; LONG, S. C.; CAIOZZO, V. J. lsokinetic torqueimbalance in the rotator cuff of the elite water polo player. The American Journal of Sports Medicine, v. 19, p. 72-5, 1992.

MONTEIRO, W. D. Medida da força muscular aspectos metodológicos e aplicações. Treinamento Desportivo, v. 3, n. 1, p. 38-51, 1998.

SAHRMANN, S. A. Diagnóstico e tratamento das síndromes de disfunção motora. São Paulo: Santos, 2005.

SOUZA, P. M. Projeto e desenvolvimento de um aparelho para avaliar a força muscular isométrica dos rotadores do ombro. 2003. 94 f. Dissertação (Pós-graduação Interunidades em Bioengenharia)-Escola de Engenharia de São Carlos, São Carlos, 2003.

TORRES, R.; GOMES, J. L. Measurement glenohumeral rotation in asymptomatic tennis players and swimmers. The American Journal of Sports Medicine, v. 37, n. 5, p. 1017-23, 2009.

TREIBER, F. A. et al. Effects theraband and lightweight dumbbel training on shoulder rotation torque and serve performace in college tennis player. The American Journal of Sports Medicine, v. 26, n. 4, p. 510-515, 1998. 